\title{
Penetration depth extraction in high temperature superconducting microwave transmission lines
}

D. Ladret, B. Cabon, J. Chilo, P. Xavier*, J. Richard* and O. Buisson*

LEMO-ENSERG, URA 833, BP. 257, 38026 Grenoble cedex, France

* CRTBT - CNRS, BP. I66X, 38042 Grenoble cedex, France

\begin{abstract}
This paper presents both modeling and measurements of the total inductance of an inverted superconducting microwave microstrip line. From the comparison between both experimental data and numerical results two of the most important parameters of the superconducting behaviour (the London's penetration depth and the critical temperature) are derived by fitting. Two superconducting physical models are then compared and are finally discussed.
\end{abstract}

\section{INTRODUCTION.}

In the last few years, a lot of significative progresses have been made in High Temperature Superconducting (HTS) technology, since thin film deposition techniques and etching methods [1] are now well known. HTS materials appear extremely promising for very fast electronic applications due to very low losses and dispersion at microwave frequency. But for accurate microwave simulations a precise superconducting modeling is required and it is necessary to exactly know basic parameters such as the critical temperature $\left(\mathrm{T}_{\mathrm{C}}\right)$ and the penetration depth at 0 Kelvin $\left(\lambda_{0}\right)$ of HTS material. In this paper, modelled data and experimental results of the total inductance of an YBCO line are presented and compared. Then, $T_{c}$ and $\lambda_{0}$ are derived from experimental data fitting.

\section{MODELING CONCEPTS.}

\subsection{Electromagnetic modeling.}

The electromagnetic modeling is based on integral Maxwell equations [2, 3] solved using the Moment Method in order to determine the distributions of current and charge density $J$ and $\rho$ respectively. Each conductor is assumed to have a uniform cross-section along the propagation axis, thus integral equations are reduced to their $2 \mathrm{D}$ expressions, $\mathrm{J}=\mathrm{J}_{\mathrm{Z}}$ and the problem is the determination of $\mathrm{J}$ and 
$\rho$ in the transversal cross-section of the line. For determining for example the current density J, using the Maxwell equations, the potential drop per unit length on an elementary filiform branch is written as :

$$
u\left(r_{i}\right)=\frac{J\left(r_{i}\right)}{\sigma_{i}}+j \omega \sum_{j=1}^{N c} \int_{s_{j}} G_{m}\left(r_{i}, r_{j}\right) J\left(r_{j}\right) d s_{j} .
$$

$N_{c}$ is the total number of conductors, $\sigma_{i}$ is the conductivity of the conductor $i$ and $G_{m}$ is the $2 D$ magnetic Green function that can be expressed by :

$$
\mathrm{G}_{\mathrm{m}}=\frac{-\mu_{0} \mu_{\mathrm{r}}}{4 \pi} \operatorname{Ln}\left|\mathrm{r}_{\mathrm{i}}-\mathrm{r}_{\mathrm{j}}\right|
$$

in which $r_{j}$ is the source position and $r_{j}$ is the observing point position (see Fig.1).

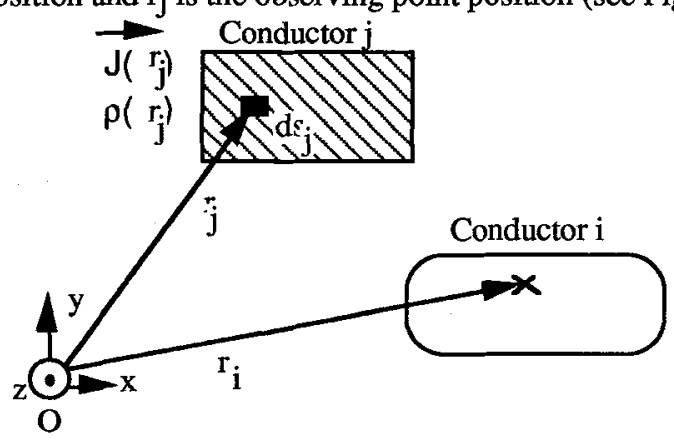

Fig. 1 ; Positions of the source and observing points.

In order to solve the integral equations the transversal cross-section of each strip is divided into elementary sections in which the current density is supposed constant. For low frequency "approximation" ( $f<10 \mathrm{GHz})$, the transversal section of the conductor $i$ is equipotential; global expressions of the potential drop (per unit length) $U_{i}$ in conductor $i$ and of the total current $I_{j}$ in conductor $\mathrm{j}$ can be calculated by :

$$
U_{i}=u\left(r_{i}\right) \text { and } I_{j}=\int_{s_{j}} J\left(r_{j}\right) d s_{j}
$$

By applying now the Moment Method $[3,4]$, the integral equation can be written in a matrix form. Then the matrices [U] (potential per unit length) and [I] of the global system of $\mathrm{N}_{\mathrm{C}}$ conductors can be related by :

$$
\left[\mathrm{U}_{\mathrm{i}}\right]=\left[\mathrm{Z}_{\mathrm{ij}}\right]\left[\mathrm{I}_{\mathrm{j}}\right]
$$

The series impedance matrix $\left(Z_{S}\right)$ and the parallel admittance matrix $\left(Y_{p}\right)$ are then obtained. For a unique line only the matrix is reduced to a scalar $Z_{S}=R+j L \omega$, where $R$ is the series resistance and $L$ is the total inductance per unit length of the line. 


\subsection{Superconducting modeling.}

The superconducting behaviour is described using alternatively the two-fluid model with the London equations and the Bipolaron equations proposed by O.Vendik [5] and used by Itoh [6].

For these cases and for $\mathrm{T}<\mathrm{T}_{\mathrm{C}}$ the conductivity used in Eq.(1) is a complex :

$$
\sigma=\sigma_{\mathrm{n}}-\mathrm{j} \sigma_{\mathrm{s}}
$$

Two different models have been used : the classical London model and the "enhanced" Bipolaron model. In the first case, using London equations, the real part of $\sigma$, related to the normal electrons, and the imaginary part, related to the super electrons are expressed by :

$$
\begin{aligned}
& \sigma_{n}(T)=\sigma\left(T_{c}^{+}\right)\left(\frac{T}{T_{c}}\right)^{4} \\
& \sigma_{s}(\omega, T)=\frac{1}{\mu \omega \lambda_{L}^{2}(T)}
\end{aligned}
$$

where $\sigma\left(T_{c}\right)$ is the normal state conductivity at the onset of the resistive transition and $\lambda_{L}(T)$ is the London penetration depth. The temperature dependence of the London penetration depth is expressed by:

$$
\lambda_{L}(T)=\frac{\lambda_{L}(0)}{\sqrt{1-\left(\frac{T}{T_{c}}\right)^{4}}}
$$

In the second case, using Bipolaron equations, alternative expressions of (6) and (8) are :

$$
\begin{aligned}
& \sigma_{\mathrm{n}}(\mathrm{T})=\sigma\left(\mathrm{T}_{\mathrm{c}}^{+}\right)\left(\frac{\mathrm{T}}{\mathrm{T}_{\mathrm{c}}}\right)^{\frac{1}{2}} \\
& \lambda_{\mathrm{L}}(\mathrm{T})=\frac{\lambda_{\mathrm{L}}(0)}{\sqrt{1-\left(\frac{\mathrm{T}}{\mathrm{T}_{\mathrm{c}}}\right)^{\frac{3}{2}}}}
\end{aligned}
$$

The two-fluid model used here is very interesting because it is simple and easy to apply in electromagnetic modeling. Moreover, the two-fluid model is not sensitive on the material structural quality but more sensitive on the basis of the superconductive phenomena. Then comparison between the two models are investigated herein.

\section{COMPARISON OF MODELING AND MEASUREMENT DATA.}

An inverted microstrip structure is used (see Fig.2), and two thin film deposition techniques investigated:

i) laser ablation (sample $R_{1}$ ) and

ii) chemical vapour deposition (MOCVD) (sample $\mathrm{T}_{3}$ ). 
Geometrical data of these two lines are given in table 1.

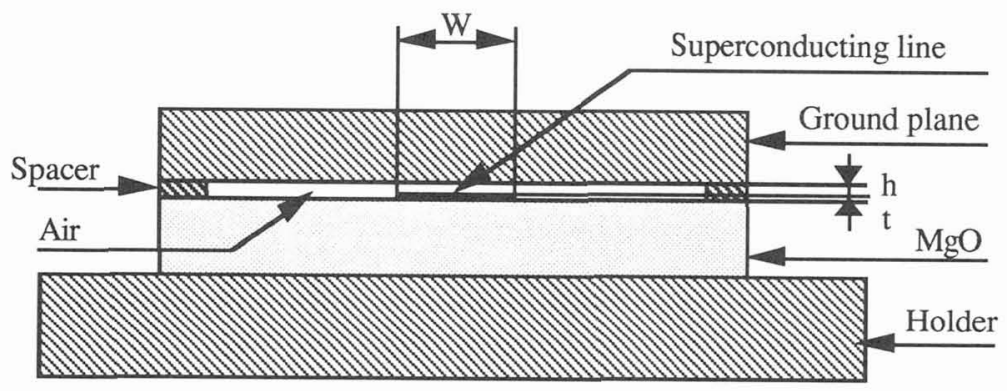

Fig. 2 : Cross-section of the experimental sample.

Table 1 : Line geometrical data.

\begin{tabular}{|c|c|c|c|c|}
\cline { 2 - 5 } \multicolumn{1}{c|}{} & $\begin{array}{c}\text { Substrate type } \\
(\mu \mathrm{m})\end{array}$ & $\begin{array}{c}\text { Air thickness } \mathrm{h} \\
(\mu \mathrm{m})\end{array}$ & $\begin{array}{c}\text { Strip width } \\
\mathrm{W}(\mu \mathrm{m})\end{array}$ & $\begin{array}{c}\text { strip thickness } \\
\mathrm{t}(\mu \mathrm{m})\end{array}$ \\
\hline $\mathrm{R} 1$ & $\mathrm{MgO} \varepsilon_{\mathrm{r}}=9.6$ & 23 & 43 & 0.13 \\
\hline $\mathrm{T} 3$ & $\mathrm{LaAlO} 3 \varepsilon_{\mathrm{r}}=25$ & 20 & 45 & 0.13 \\
\hline
\end{tabular}

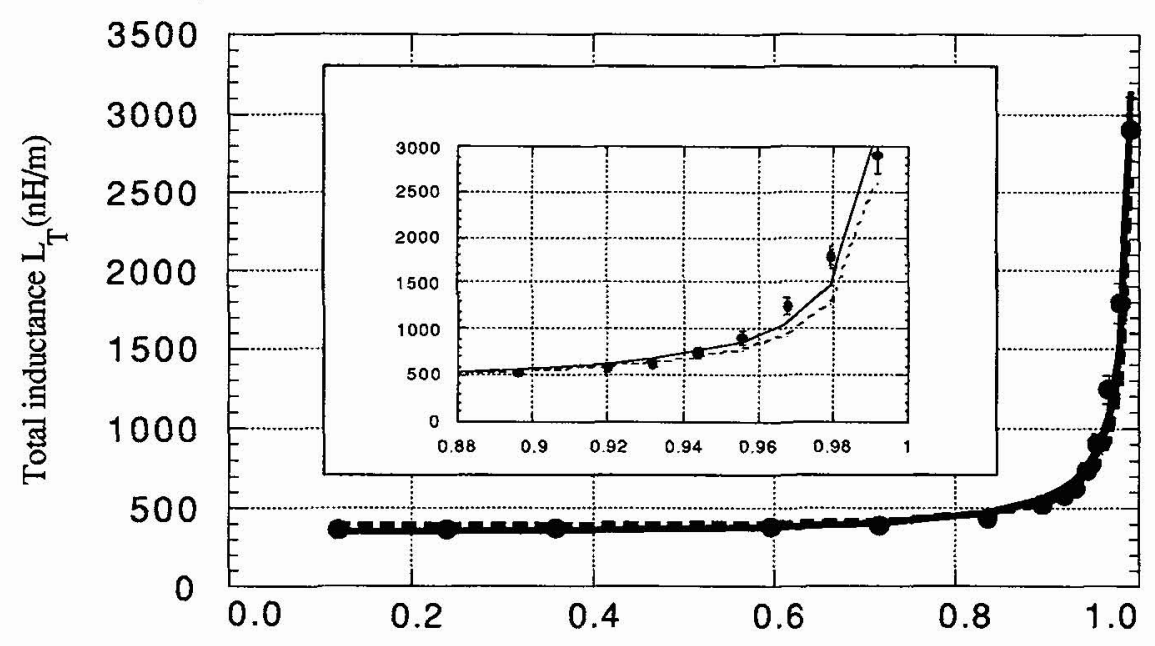

Fig.3a : Experimental versus modeled results for sample $T_{3}$.

Modeled results with "enhanced" two-fluid model

. . . - Modeled results with the two-fluid model and London's equation

- Experimental data 


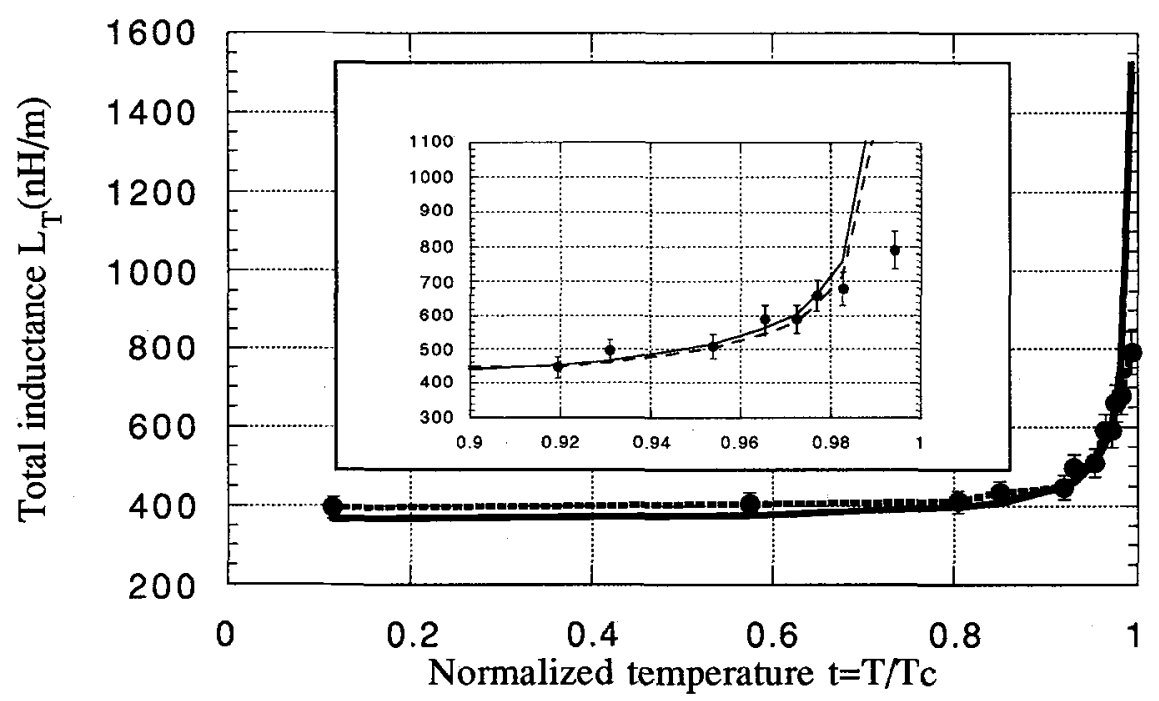

Fig.3b : Experimental versus modeled results for sample $\mathbf{R}_{1}$.

Modeled results with "enhanced" two-fluid model

- Modeled results with the two-fluid model and London's equation

- Experimental data

The critical temperature $T_{c}$ is taken as the temperature where the resistivity begins to climb up. From a four probe measurement of the $\mathrm{DC}$ resistivity, the estimated critical temperature is $\mathrm{T}_{\mathrm{c}}=87 \pm 0.1 \mathrm{~K}$ for sample $R_{1}$ and $T_{C}=84 \pm 0.5 \mathrm{~K}$ for sample $T_{3}$. Fig.3a for sample $T_{3}$ and Fig. $3 b$ for sample $R_{1}$ show the comparison of the total inductance variations with temperature obtained from experimental results and modeling. Also, London equations and Bipolaron equations are compared on the fitting curves.

The two models are in good agreement with experimental results and two corresponding sets of parameters (penetration depth and critical temperature) are derived and indicated in table 2.

Table 2 : Fitting results.

\begin{tabular}{|c|c|c||c|c|}
\cline { 2 - 5 } \multicolumn{1}{c|}{} & \multicolumn{2}{c||}{ London model } & \multicolumn{2}{c|}{ Bipolaron model } \\
\cline { 2 - 5 } \multicolumn{1}{c|}{} & $\lambda_{\mathrm{L}}(0)(\mu \mathrm{m})$ & $\mathrm{T}_{\mathrm{C}}(\mathrm{K})$ & $\lambda_{\mathrm{L}}(0)(\mu \mathrm{m})$ & $\mathrm{T}_{\mathrm{C}}(\mathrm{K})$ \\
\hline $\mathrm{R}_{1}$ & 0.32 & 87.0 & 0.21 & 87.0 \\
\hline $\mathrm{T}_{3}$ & 0.59 & 83.7 & 0.40 & 83.7 \\
\hline
\end{tabular}

For the best fit of the experimental data, the critical temperature must be considered as a modeling parameter varying within the measurement precision. The most important unknown parameter is the penetration depth $\lambda_{\mathrm{L}}(0)$ at $0 \mathrm{Kelvin}$, and is also derived from fitting. But, this parameter depends on the superconducting physical model used. Consequently, an absolute result of $\lambda_{0}\left(=\lambda_{L}(0)\right)$ can not be 
provided but only an approximated value according to the model used and it can be noticed that the London model gives higher penetration depth than the Bipolaron model. But comparing with the values of $\lambda_{\mathrm{L}}(0)$ obtained on bulk materials, the Bipolaron model seems to give better results.

\section{CONCLUSION.}

The originality of this work is to propose a new method for extracting the London's penetration depth $\lambda_{\mathrm{L}}(0)$ at $0 \mathrm{Kelvin}$. The comparison between both modeling data and experimental results validates the electromagnetic concept. Simulations use both the classical model of London and the bipolaron model but the more suitable model of HTc superconducting thin film can not be determined from these results.

\section{REFERENCES :}

[1] P. Xavier, T. Fournier, J. Chaussy, J. Richard and M. Charalambous, J. Appl. Phys. 75 (1994) 1219-1221.

[2] R. F. Harrington, C. Wei, IEEE Trans. Microwave Theory Tech. 32 (1984) 705-710.

[3] A. Amadouche, J. Chilo, IEEE Trans. Comp. Hybrid. Manuf. Technol. 12 (1989) 124-129.

[4] R.F. Harrington, Field Computation by Moment Methods, (Krieger Publishing Co. Melbourne, 1989) Chapter 7.

[5] O.G. Vendik, Soviet Technical Physics Letters 14 (1988) 482.

[6] O. R. Baiocchi, K. S. Kong, T. Itoh, IEEE Trans. Microwave Theory Tech. 40 (1992) 509-514. 